\title{
Análisis crítico de un artículo: ¿Es confiable la medición de adenosin deaminasa (ADA) para el diagnóstico de derrame pleural tuberculoso?
}

Cristian Herrera $R^{1 a}$, Carlos Aravena $L^{1,2,3}$.

Critically appraised article: Diagnostic accuracy of adenosine deaminase in tuberculous pleurisy: A metaanalyses Respir Med 2008; 102: 744-54. Q iu-Li Liang, Huan-Zhong Shi, Ke Wang, Shou-Ming Q in, Xue-Jun Q in.

Background: Conventional tests are not always helpful in making a diagnosis of tuberculous pleurisy. Many studies have investigated the usefulness of adenosine deaminase (ADA) in pleural fluid for the early diagnosis of tuberculous pleuresy. We conducted a metaanalysis to determine the accuracy of ADA measurements in the diagnosis of tuberculous pleurisy. Methods: After a systematic review of English language studies, sensitivity, specificity, and other measures of accuracy of ADA concentration in the diagnosis of pleural effusion were pooled using random effects models. Summary receiver operating characteristic curves were used to summarize overall test performance. Results: Sixty-three studies met our inclusion criteria. The summary estimates for ADA in the diagnosis of tuberculous pleurisy in the studies included were sensitivity 0.92 (95\% confidence interval 0.90-0.93), specificity 0.90 (95\% confidence interval 0.89-0.91), positive likelihood ratio 9.03 (95\% confidence interval 7.19-11.35), negative likelihood ratio 0.10 (95\% confidence interval 0.07-0.14), and diagnostic odds ratio 110.08 (95\% confidence interval 69.96-173.20). Conclusions: ADA determination is a relative sensitive and specific test for the diagnosis of tuberculous pleurisy. Measurement of ADA in pleural effusion is thus likely to be a useful diagnostic tool for tuberculous plerisy. The results of ADA assays should be interpreted in parallel with clinical findings and the results of conventional tests.

\footnotetext{
${ }^{1}$ Unidad de Medicina Basada en Evidencia.

${ }^{2}$ Departamento de Medicina Interna, Pontificia Universidad Católica de Chile. Santiago de Chile.

${ }^{3}$ Servicio de Medicina, Hospital Dr. Sótero del Río.

anterno de Medicina, Facultad de Medicina, Pontificia Universidad Católica de Chile.
}

Correspondencia a: Dr. Carlos Aravena. E mail: caaraven@uc.cl 


\section{Conclusión de LOS Revisores:}

La medición de adenosin deaminasa (ADA) es un test relativamente sensible y específico para el diagnóstico de un derrame pleural tuberculoso. Los resultados deben ser interpretados en relación a los hallazgos clínicos y los resultados de tests convencionales.

\section{PREgunTA CLÍNICA:}

En pacientes con sospecha de derrame pleural tuberculoso, ¿es la medición de ADA en el líquido pleural, confiable para confirmar el diagnóstico?

\section{Contexto:}

La tuberculosis (TBC) es una enfermedad infecciosa de alto impacto a nivel mundial. En Chile, su incidencia es de 15 casos por 100.000 habitantes al año y la letalidad de 1 por 100.000 habitantes al año, mientras en Perú es 162 y 16, en Brasil 50 y 4, en España 30 y 3, y en Canadá 5 y 1 , respectivamente ${ }^{1}$.

El derrame pleural tuberculoso (DPT) es causado por una reacción de hipersensibilidad severa de tipo retardado, en respuesta a la ruptura de focos subpleurales de la infección por el Mycobacterium tuberculosis. El proceso inflamatorio resulta en un aumento en la permeabilidad vascular pleural llevando a la acumulación de un fluido rico en proteínas y al reclutamiento de leucocitos en el espacio pleural ${ }^{2}$. Se observa en $5 \%$ de los pacientes con TBC constituyendo la segunda forma más común de tuberculosis extra-pulmonar ${ }^{3}$.

El diagnóstico definitivo del DPT depende de demostrar la presencia del M tuberculosis en la expectoración, el líquido pleural o en una biopsia pleural.

Hallazgos que apoyan el diagnóstico son la presencia de granulomas tuberculosos en la pleura, niveles elevados de interferón gamma y de ADA en el líquido pleural ${ }^{3}$. La confiabilidad de la ADA pleural para el diagnóstico de DPT ha sido investigada en muchos estudios. Dos metaanálisis han mostrado su utillidad ${ }^{4,5}$, pero sus "Likelihood ratios" no han sido evaluados. Si bien otros tests como la medición de IFN-gamma ${ }^{6}$, han demostrado ser mejores que la ADA para el diagnóstico de DPT, en India, se estimó que en países de bajo y mediano ingreso (como Chile) el uso de ADA es más costo-efectivo que la medición de IFN-gamma para diagnosticar $\mathrm{DPT}^{7}$. Dado que no existen biomarcadores específicos para DPT y que los médotos clásicos de diagnóstico de la TBC son invasivos y presentan limitaciones para el diagnóstico de DPT, conocer las características de un test simple como el ADA tiene gran relevancia clínica.

\section{Características del estudio:}

Tipo de estudio: Revisión sistemática de estudios de diagnóstico. La búsqueda se realizó en: Embase, Web of Science, Cochrane Database y Medline. Se buscaron estudios publicados en inglés hasta marzo de 2007. También se realizó una búsqueda manual de las referencias de los estudios encontrados y se contactó a autores y a expertos en el área.

Se incluyeron estudios con más de 10 pacientes con posible DPT, si entregaban la sensibilidad y especificidad del ADA para el diagnóstico del DPT o si entregaban los datos para calcularlo (por ej: en "dot plots"). Con esto, se calculó una curva ROC (Receiving Operating Characteristic) individual para cada estudio y luego se confeccionó un SROC 
(Summary ROC). Se usó el modelo de efectos aleatorios para calcular los estimadores puntuales combinados de la sensibilidad, especificidad, likelihood ratio positivo (LRP), likelihood ratio negativo (LRN) y Odds ratio de Diagnóstico (ORD).

VALIDEZ:

Pregunta específica y focalizada

Búsqueda amplia y completa

Criterios de inclusión explícitos y adecuados

Evaluación de calidad de los estudios incluidos

Reproducibilidad del proceso

Evaluación de heterogeneidad
Sí

Parcialmente*

No

$\mathrm{S}^{* * *}$

Sí

Sí

* Sólo se incluyeron estudios en idioma inglés, no se buscó "literatura gris".

** Se realizó una evaluación de la calidad mediante los métodos siguientes: STARD

(Standards for Reporting Diagnostic Accuracy) y QUADAS (Quality Assessment for Studies of Diagnostic Accuracy).

\section{Resultados:}

Se incluyeron 63 estudios. En total, 2.796 pacientes tuvieron un resultado positivo y 5.297 negativo para DPT. La medición de ADA se realizó con método Giusti en 42 estudios, con otro método en 17 y 4 no reportaron el método usado. Como Gold Standard (GS) de DPT, 28 estudios consideraron criterios bacteriológicos o histológicos (GS ideal); 34 reportaron un "gold standard" no enunciado o el curso clínico, o análisis del líquido pleural, o radiología o respuesta a terapia anti-tuberculosa; 1 estudio no mencionó uso de GS.

Los puntos de corte de los estudios incluyendo transudados ( $n=34)$ y los que no los incluyeron $(\mathrm{n}=20)$ fueron $43,1 \pm 1,6 \mathrm{UI} / \mathrm{L}$, respectivamente, sin diferencia estadística $(\mathrm{p}$ $=0,125$ ). Además, el punto de corte utilizando el método Giusti ( $n=42,44,0 \pm 1,4 \mathrm{UI} / \mathrm{L}$ ) fue mucho más que los que utilizaron otros métodos ( $n=17,38,1 \pm 2,3 \mathrm{UI} / \mathrm{L}, \mathrm{p}=0,034)$.

Se evaluó heterogeneidad con $\mathrm{Chi}^{2}$ y el test exacto de Fisher. Hubo heterogeneidad significativa entre los estudios $(\mathrm{p}<0,01)$.

Tabla 1. Resultados de la revisión sistemática

\begin{tabular}{|lcccc|}
\hline Test & $\begin{array}{c}\text { Sensibilidad } \\
(\text { IC 95\%) }\end{array}$ & $\begin{array}{c}\text { Especificidad } \\
\text { (IC 95\%) }\end{array}$ & $\begin{array}{c}\text { LRP } \\
(\text { IC 95\%) }\end{array}$ & $\begin{array}{c}\text { LRN } \\
(\text { IC 95\%) }\end{array}$ \\
\hline $\begin{array}{l}\text { ADA en líquido } \\
\text { pleural }\end{array}$ & $\begin{array}{c}0,92 \\
(0,90-0,93)\end{array}$ & $\begin{array}{c}0,90 \\
(0,89-0,91)\end{array}$ & $\begin{array}{c}9,03 \\
(7,19-11,35)\end{array}$ & $\begin{array}{c}0,10 \\
(0,07-0,14)\end{array}$ \\
\hline
\end{tabular}

$\begin{array}{lll}\mathrm{LRP} & = & \text { Likelihood Ratio resultado ADA Positivo }(>40) \\ \mathrm{LRN} & = & \text { Likelihood Ratio resultado ADA Negativo }(\leq 40) \\ \mathrm{IC} & = & \text { Intervalo de confianza }\end{array}$




\section{COMENTARIOS Y APLICACIÓN PRÁCTICA:}

1. Este estudio cumple parcialmente con los criterios de validez de una revisión sistemática, lo que introduce un alto riesgo de sesgo:

a. La búsqueda utilizó varias bases de datos, pero sólo de estudios en inglés y no se buscó en la "literatura gris", lo que puede producir sesgo de publicación. Considerando que la mayor prevalencia de TBC ocurre en países en vías de desarrollo, donde no predomina el idioma inglés y que estos posibles estudios no se publican en revistas indexadas, existe alta probabilidad de que no se hayan incluido estudios relevantes, lo que es apoyado por el "Funnel plot" asimétrico (test estadístico de evaluación de sesgo de publicación) de este estudio.

b. Los criterios de inclusión y exclusión no son claros, no define bien el tipo de pacientes, de métodos diagnósticos, ni el diseño del estudio que se debía incluir.

c. La calidad metodológica de los estudios incluidos es modesta en la mayoría de ellos.

2. Además, la heterogeneidad en esta revisión sistemática resultó significativa, lo que probablemente esté explicado por los distintos tipos de pacientes, diferentes métodos diagnósticos, puntos de corte y patrones de referencia utilizados (esto último produce también sesgo de verificación). Ello resta validez al metaanálisis realizado, haciendo difícil interpretar sus resultados.

3. La aplicabilidad de este estudio es difícil de evaluar, porque no está claro el tipo de pacientes seleccionados y se mezclan distintos métodos de medición del ADA en líquido pleural y diferentes puntos de cortes.

4. En suma, si bien los resultados muestran que valores de ADA $>$ y $<40$ aumentarían o disminuirían respectivamente en aproximadamente 10 veces la probabilidad de TBC, el estudio tiene un alto riesgo de sesgo, por lo que sus resultados se deben interpretar con extrema cautela.

\section{REFERENCIAS}

1. Global Tuberculosis Database. World Health Organization, 2008. Disponible en: http:// www.who.int/globalatlas/dataQuery/default.asp [Consultada el 14 de mayo, 2008].

2. Chakrabarti B, Davies PDO. Pleural tuberculosis. Monaldi Arch Chest Dis 2006; 65: 26-33.

3. Gopi A, Madhavan SM, Sharma SK, Sahn SA. Diagnosis and Treatment of Tuberculous Pleural Effusion in 2006. Chest 2007; 131: 880-89.

4. Goto M, Noguchi Y, Koyama H, Hira K, Shimbo T, Fuku T. Diagnostic value of adenosine deaminase in tuberculous pleural effusion: a metaanalysis. Ann Clin Biochem 2003; 40: 374-81.

5. Greco S, Girardi E, Masciangelo R, Capoccetta GB, Saltini C. Adenosine deaminase and interferon gamma measurements for the diagnosis of tuberculous pleusery: a metaanalysis. IntJ Tuberc Lung Dis 2003; 7: 777-86.

6. Jiang J, Shi HZ, Liang QL, QIN SM, Qin XJ. Diagnostic value of interferon-gamma in tuberculous pleurisy: a metaanalysis. Chest 2007; 131: 1133-41.

7. Sharma SK, Banga A. Pleural fluid interferon-gamma and adenosine deaminase levels in tuberculosis pleural effusion: a cost-effectiveness analysis. J Clin Lab Anal 2005; 19: 40-6. 